\title{
THEORY OF GALACTIC NUCLEI
}

\author{
WILLIAM C. SASLAW \\ Dept. of Astronomy and Center for Advanced Studies, University of Virginia, \\ National Radio Astronomy Observatory, Charlottesville, Virginia and \\ Institute of Astronomy, Cambridge Mass., U.S.A.
}

We dance around in a ring and suppose, But the Secret sits in the middle and knows.

ROBERT Frost

\begin{abstract}
This paper reviews current ideas about the possible constituents of galactic nuclei, and the mechanisms for ejecting gas and massive objects.
\end{abstract}

We come now to the very innermost regions of a galaxy. What generates the enormous energies that pour from galactic nuclei? How do they evolve? And are new physical laws needed to understand them? Even though I've only asked three questions so far, already the ratio of questions to answers is infinite! At present there is no complete, comprehensive and compelling theory of galactic nuclei. Rather, there are a number of approaches, each emphasizing different major constituents of galaxies. It is likely that several of these constituents, and perhaps some not yet imagined, will play important parts in our eventual understanding of galactic nuclei.

By the nucleus, I mean the region where most of the violent action is. In many galaxies this has a radius less than $\sim 10 \mathrm{pc}$, and it may be very much less. There is no shortage of possible explanations for all the radio and optical activity we've recently heard about. Rather, the main problem is to find definitive tests of these proposals. The difficulty, as we will see, is that galactic nuclei can be very complex objects with a rich array of physical phenomena. Thus we should be careful not to be over-simple in our picture of galactic nuclei. On the other hand, we should also keep in mind the syndrome paraphrased from Ecclesiastes: Of the making of many models there is no end, and too many parameters are a weariness of the flesh.

In this review I will try to describe the current state of our understanding of the basic physics underlying galactic nuclei. Although model-building is certainly important to progress in this field, I won't say much about the virtues or disadvantages of particular models, except as occasional illustrations which are related fairly directly to observations. Many of these observational connections would improve significantly in the next decade if statistical properties of nuclei become better known. Our main problem is that we don't know how typical the present observations are, in any reasonably rigorous sense. These observations are a bit impressionistic. There is a danger in biasing general theories toward a few well-studied cases which may not be very representative of active galactic nuclei. I'll try also to emphasize results since the subject was last reviewed in 1970 at the Vatican Symposium (O'Connell, 1971) and by Burbidge (1970). The general picture of the evolution of galactic nuclei as dense stellar systems is reviewed in detail elsewhere (Saslaw, 1973) so I won't spend much 
time on it here. The scope of this review is broader, but less detailed, than the one of dense stellar systems.

Let us start by considering, in turn, the major constituents of galactic nuclei. These fall into two classes: Those observed to exist in galactic nuclei, and those observed to exist in the minds of theoreticians. The first class contains:

(1) electromagnetic radiation, (2) gas, (3) dust, and (4) stars;

and the second class contains:

(5) supermassive objects, (6) rotating magnetoids, (7) pulsar systems, (8) singularities in general relativity, (9) accretion disks, (10) anti-matter, (11) gravitational radiation, and (12) new physics.

We discuss first the general physical properties of these objects and their relation to some observational questions such as the spectra, variability, and lifetimes of galactic nuclei. Then we use some of these constituents to review one question of current interest in more detail: How can galactic nuclei eject large amounts of matter?

\section{Constituents of Galactic Nuclei}

\subsection{EleCtromagnetic RADiATION}

The optical, radio, and infrared observations reviewed by Drs Ulrich (p. 279) and Ekers (p. 257) provide direct evidence for intense electromagnetic radiation in galactic nuclei. The small sizes of the radio emitting volumes are found from very long baseline measurements with resolution $\sim 4 \times 10^{-4}$ arc sec at $\lambda=6 \mathrm{~cm}$ (Kellermann et al., 1971). Many sources, both quasars and galaxies, have strong radio components less than several parsecs in diameter. In Centaurus A (NGC 5128) Kunkel and Bradt (1971) have detected an infrared hot spot with a size of about $75 \times 110 \mathrm{pc}$ and a total luminosity of $\sim 2 \times 10^{41} \mathrm{erg} \mathrm{s}^{-1}$. They identify this as the nucleus of the galaxy. Within this nucleus is a non-thermal radio source (Wade et al., 1971) whose linear size is measured to be less than $13 \mathrm{pc}$. If this is synchrotron radiation which is self-absorbed at centimetric wavelengths the size of the emitting region is only about one light year.

Photometry between 2 and $25 \mu$ shows that the infrared luminosity of galaxies ranges from $10^{37}$ to $\sim 10^{45} \mathrm{erg} \mathrm{s}^{-1}$. In many cases, and especially for Seyferts, the infrared losses dominate the total luminosity of the galaxy. Unfortunately the lower limits on the size of the emitting regions are usually much greater than for the radio regions. Thus far, detailed comparisons of the locations of radio, infrared, and optical hot spots in galactic nuclei have been made only for the Milky Way (Downes and Martin, 1971) and the exploded galaxy M82 (Kronberg et al., 1972). In neither case is there any detailed correspondence between the positions of strong infrared and radio regions. However, these are not typical strongly concentrated nuclei, and many more observations of this type will be useful for understanding the radiation mechanisms.

In the optical, the smallest upper limit to the size of a Seyfert nucleus comes from the Stratoscope II photograph of NGC 4151 (Schwarzschild, 1973). The photographs taken on the seventh Stratoscope flight had higher definition than those of the previous flight. From the earlier flight, an upper limit to the half-intensity diameter of the 
nucleus of NGC 4151 was $\sim 1 " 7$. The new measurement reduced this by about a factor of two. However, in the interval between the two observations, the value of the Hubble constant also decreased by a factor of about two, leaving the upper limit to the linear size of the nucleus much the same. The upper limit for the half-power radius is now $3.5 \mathrm{pc}$ and the luminosity, mostly non-thermal, is $\sim 2 \times 10^{43} \mathrm{erg} \mathrm{s}^{-1}$.

There appear to be some relations between the optical, infrared, and radio emission of galactic nuclei. In spiral galaxies, there is a strong tendency for compact radio sources to be present if the nucleus is starlike, and especially if the nucleus is split into two or more unresolved components (Tovmassian, 1972). For elliptical galaxies there is not yet much information. Some ellipticals have sharp central peaks of optical luminosity, while others are smoother. Although the compact active radio ellipticals usually have optical emission lines (Disney and Cromwell, 1971), their correlation with central optical luminosity peaks is not established (Heeschen et al., 1971). One relation which is better established, however, is that the radio luminosity of Seyfert or related galaxies is usually proportional to its infrared luminosity (van der Kruit, 1971; Rieke and Low, 1972a).

Thus there is evidence for strong radiation in galactic nuclei. The intense pressure of this radiation may drive gas out of the system. In the simplest case when the pressure is caused by resonance line scattering in an optically-thin spherically-symmetric medium, the radiative acceleration due to a species $i$ is (Mushotzky et al., 1972)

$$
g_{\mathrm{r}}(i)=\stackrel{F_{v}}{c} \frac{N_{\mathrm{a}}}{N_{\mathrm{H}}} \frac{N_{\mathrm{i}}}{N_{\mathrm{a}}} \frac{X}{m_{\mathrm{H}}} m_{\mathrm{e}} c f .
$$

Here, $F_{v}$ is the continuum flux at the line centre, $N_{\mathrm{H}}, N_{\mathrm{a}}$, and $N_{\mathrm{i}}$ are respectively the number densities of hydrogen (which has mass fraction $X$ ), of the element, and of the relevant ionization state, $f$ is the $f$-value of the transition, and the other physical constants have their usual meaning. If the scattering is not from a resonance line, the left-hand side of Equation (1) is multiplied by the probability of finding an ion in the relevant excited state. When the only radial dependence in $g(r)$ comes from the flux, or if all the factors fortuitously give $g(r) \sim r^{-2}$, then both radiative and gravitational accelerations of the gas have the same spatial dependence. Thus there will be a critical mass which can produce a static situation where gravity balances radiation pressure. More mass makes the gas fall in, less mass lets it blow out. In general, however, the situation will be too complicated to be in balance and the results will depend on details of the model.

One important aspect of radiation-driven mass loss is less model-dependent. This is a 'line locking' mechanism which can produce narrow velocity ranges for the outflowing gas. Originally suggested nearly $50 \mathrm{yr}$ ago by Milne (1926) to produce solar cosmic rays, 'line locking' is now being revived as a possible explanation for narrow absorption lines in quasars, stars and Seyfert nuclei (Scargle, 1973; Williams, 1972; De Young et al., 1973). The basic idea is that gas in the nucleus produces a continuum with strong absorption lines. Filaments of gas near, or outside the edge of, the nucleus are accelerated by gravity and by radiation pressure in the absorption line. If the bal- 
ance between these two accelerations is destroyed and the filaments increase their velocity, they will begin to absorb the more intense radiation from the blue wing of the line and eventually, as the velocity increases still further, the filaments absorb from the strong continuum and are driven out of the galaxy. Similarly, infalling filaments would be decelerated.

There are several ways in which these filaments may reach a definite line-locked limiting velocity: (a) if there is only one dominant absorption line, the filament will be accelerated by the continuum and its final velocity will depend on the depth of the line as well as the size and luminosity of the accelerating region, (b) if there are two strong absorption lines, the filament is accelerated along the continuum until its absorption is blueshifted to the second line, at which point the radiation pressure drops and the filament achieves a limiting velocity equivalent to the separation of the two lines, (c) if a strong absorption line is located to the red of the Lyman continuum drop, the filament can similarly be accelerated to a velocity equivalent to the difference between the line and the Lyman drop. For a filament to be accelerated coherently by radiation pressure, photons created within the filament must not be absorbed inside it (Williams, 1972). The simplest condition this implies is that, when the filaments are accelerated, they are optically thin in the absorption line. We will return to this effect of radiation in our discussion of the ejection of matter from galactic nuclei. Radiation pressure can be especially important if the brightness temperature at low frequencies, $T_{\mathrm{b}}$, exceeds the rest mass of the electron, for then induced Thomson scattering becomes important and may increase the temperature of the electrons to nearly $T_{\mathrm{b}}$, and distort the radiation spectrum (Levich et al., 1972).

\subsection{GAS}

Clouds of gas in galactic nuclei have been detected from their radiation in Balmer lines, their emission in the forbidden lines of nitrogen, oxygen and other heavy elements, and in $21-\mathrm{cm}$ absorption. In two galaxies, 3C 390.3 (Burbidge and Burbidge, 1971) and NGC 1275 (Burbidge and Burbidge, 1965) the emission lines show two systems of redshifts, suggesting that gas is being expelled from the system. In other cases, such as the Seyfert I $4329 \AA$, there is evidence for high-velocity motion of the gas in the nucleus, at velocities up to $\sim 13000 \mathrm{~km} \mathrm{~s}^{-1}$ (Disney, 1973).

During the last few years, people have studied a variety of fairly detailed models to account for the spectra of galactic nuclei and quasars. In addition to constraints from the observed spectrum, ranging from radio to $\gamma$-rays, the models are strongly constrained by intensity fluctuations observed over periods of days to years (e.g. Pacholczyk, 1970; Penston et al., 1971). Among the physical processes which have been invoked to reproduce the general form of the spectrum are thermal radiation by gas and dust, electron and proton synchrotron emission, inverse Compton scattering, induced Compton scattering, heating of gas by X-ray sources or by intense low frequency radiation from pulsars or spinars, scattering of photons by non-thermal plasmons, non-thermal emission from the surfaces of massive objects, and collisions of large gas clouds. Recent discussions of these processes may be found in Jones and 
Kellogg (1972), Davidson (1972), Bergeron and Salpeter (1973), MacAlpine (1972), de Sabbata et al. (1972), Ozernoy (1973), Levich and Sunyaev (1971), Arons et al. (1974), Daltabuit and Cox (1972).

Various combinations of these models can account, in a fairly consistent manner, for most observed properties of gas and radiation in galactic nuclei. While this may not be too surprising, in view of the large number of possible geometries and physical parameters, it does indicate that there is no compelling need to invoke completely new kinds of physics to explain these particular phenomena.

These models are also important for estimating the basic physical parameters of galactic nuclei, and their uncertainties. For example, a simple model of NGC 1068 (Bergeron and Salpeter, 1973) in which most of the infrared radiation is synchrotron and the X-rays are from inverse Compton scattering on the infrared, gives a radius of the active region between $10^{-2}-10^{-4} \mathrm{pc}$, a magnetic field between about $10-10^{3} \mathrm{G}$, an electron density of about $10^{2}-10^{6} \mathrm{~cm}^{-3}$ and a thermal gas density of $\sim 10^{11} \mathrm{~cm}^{-3}$. The ranges in these values are due to the assumed ratio of X-ray to infrared luminosity, between $10^{-1}$ and 10. Bergeron and Salpeter have also constructed a simple model of this. Seyfert galaxy in which the infrared is produced by thermal grains, and the $\mathrm{X}$-rays by inverse Compton scattering of the infrared. For this the emitting region must be much larger, between $10^{2}-5 \times 10^{4} \mathrm{pc}$, the electron density much less, between $6 \times 10^{-4}-1 \mathrm{~cm}^{-3}$, the number of grains between $10^{-14}-3 \times 10^{-10}$ and the total mass of grains between $5 \times 10^{4}-2 \times 10^{9} M_{\odot}$. Again this range is for the same assumed ratio of X-ray to infrared luminosities. The large size of the infrared emitting region in dust models makes it difficult for them to account for rapid luminosity fluctuations.

The stability of gas in the nucleus is an unsettled fundamental problem. There are two main possibilities: either the gas may be unstable to the formation of stars or massive objects near the centre, or it may be blown out in a galactic wind. Some time ago Arny (1970) argued that the gas in galactic nuclei would be thermally unstable and form clouds. The size of these clouds depends strongly on the manner of heating the gas, for which only the supersonic passage of stars through the gas and the deposition of energy from stellar winds into the surrounding gas were considered. Heating by $\mathrm{X}$-rays, ultraviolet, or low energy cosmic rays could change the picture considerably, and it would be useful to know how they affect the size and mass of the clouds.

Gravity may cause these clouds to collapse and fragment. It is likely that both turbulence in the cloud (Arny, 1971) and the presence of the background gravitational field of stars in the nucleus (Mathews, 1972) encourage the formation of more massive stars. Objects of several hundred solar masses could possibly form, although this is highly uncertain.

If the gas which stars shed in the nucleus is further heated to temperatures $\gtrsim 10^{6} \mathrm{~K}$ by shocks from supernovae explosions and photo-ionization, it may be expelled from the nucleus as a galactic wind (Burke, 1968; Johnson and Axford, 1971; Mathews and Baker, 1971; Wolfe, 1974). Unlike the situation in the solar wind, the sources of the gas and the gravitational field are distributed throughout the region where the wind forms instead of being a single compact body. As with the solar wind, the early 
solutions were for idealized spherically-symmetric steady flow of a perfect gas. The later solutions added enough details of heating and cooling to produce unsteady flows and possible thermal instabilities.

The most detailed models are those of Mathews and Baker. As an example, they consider a hot wind of $2 \times 10^{6} \mathrm{~K}$, fed by a stellar mass-loss rate of $3.4 \mathrm{M}_{\odot} \mathrm{yr}^{-1}$ in an elliptical galaxy with total mass $9 \times 10^{10} M_{\odot}$ and a stellar density of $440 M_{\odot} \mathrm{pc}^{-3}$ at its centre. Starting with no initial gas, a steady-state wind establishes itself in $\sim 5 \times 10^{7} \mathrm{yr}$. This is basically the time it takes gas to flow from the centre to the edge of the galaxy $(14 \mathrm{kpc})$. The mass involved in the steady wind is $\sim 8 \times 10^{7} M_{\odot}$. It starts moving subsonically at $\sim 100 \mathrm{~km} \mathrm{~s}^{-1}$ in the nucleus, and becomes supersonic in the outer parts of the galaxy, reaching $\sim 1000 \mathrm{~km} \mathrm{~s}^{-1}$ at $\sim 15 \mathrm{kpc}$. Such a wind would not be observable directly, though it could carry radiating dust along as a marker. On the other hand, if the gas is cooler $\left(\lesssim 10^{4} \mathrm{~K}\right)$, the wind is unsteady. This may occur when the central gas density rises and cooling becomes more effective, or if the supernova rate is small. Now the gas falls in toward the centre of the galaxy, perhaps to form new stars or massive objects related to the strong radio sources associated with $\sim 10 \%$ of giant elliptical galaxies.

Wolfe has shown that a hot wind flowing out from the nucleus can be thermally unstable. If so, it may form a two-phase system in which cool dense clouds are in pressure equilibrium with the hotter, more tenuous intercloud gas. These clouds would then be borne outward by the wind. If observed between us and a central continuum source, their spectra could show the narrow lines seen in some quasars and Seyfert galaxies. It is, however, difficult to use nuclear winds to produce clouds moving out from quasars at very high velocities or, in Seyferts, to produce large central clouds which do not contain a continuum source. Details of all these models are tested by computing line strengths and shapes for highly ionized atoms, especially iron. Usually it is possible to find a particular structure of the gas and radiation field which is reasonably consistent with observations.

Magnetic fields are often associated with the ionized gas in galactic nuclei. Relativistic electrons interacting with these fields produce synchrotron radio and possibly infrared and optical emission. A tell-tale sign of synchrotron radiation is its circular polarization. For an isotropic array of pitch angles, the fractional polarization is approximately $10^{-2}(\lambda B)^{1 / 2}$ where $\lambda$ is the wavelength in centimetres and $B$ the magnetic field in gauss (Sciama and Rees, 1967). Several radio sources have measured circular polarizations of a few tenths of one percent in the radio, indicating magnetic fields of $\lesssim 1 \mathrm{G}$. The degree of circular polarization can be reduced, however, if the synchrotron radiation is Compton scattered to higher energies by the same electrons that made it (Bonometto and Saggion, 1973). Thus the lack of circular polarization in the observed non-thermal optical emission (Landstreet and Angel, 1972) does not preclude the optical being produced from synchrotron radiation by inverse Compton scattering.

\subsection{Dust}

Dust is often seen in reddened Seyfert and other nuclei. Another observational reason 
for introducing dust into galactic nuclei was to account for the high infrared luminosity observed in many Seyferts and quasars (Rees et al., 1969). While thermal emission from grains at different temperatures can explain the steep spectrum observed between 2.2 and $22 \mu$, it runs into difficulty with the rapid time fluctuations observed in some sources. 3C 273 fluctuates especially violently, changing its $10 \mu$ luminosity by $10^{12} L_{\odot}$ - nearly a factor of 2 - in two months, and there is also evidence for substantial $10 \mu$ variability in NGC 1068 and NGC 4151 on time scales of weeks to years (Rieke and Low, 1972b).

To obtain thermal infrared emission by irradiating grains from a central UV source requires dust temperatures between about 10-1000 K. At these temperatures, the sizes of dusty disks needed to produce the high infrared luminosities are greater than the light travel distances during the period of fluctuation. Thus large amplitude fluctuations would not be produced. While it may be possible to diminish this incompatibility by clever modelling, the variations found recently by Rieke and Low (1972b) are inconsistent with straightforward models (Kaneko et al., 1972). However, grains could be an important contribution to the weaker $\left(L \sim 10^{42} \mathrm{erg} \mathrm{s}^{-1}\right)$ infrared emitted in the centre of our own Galaxy (Bergeron and Salpeter, 1973; Krishna Swamy, 1971), since this comes from a relatively large region of $\sim 175 \mathrm{pc}$.

\subsection{Stars}

Although the direct evidence for stars in galactic nuclei is not as compelling as the evidence for radiation and gas, there seems to be little doubt that they are present. The difficulty in determining stellar content arises mainly because the angular sizes of galactic nuclei are so small and because the non-stellar continuum and light emission lines would overpower any stellar absorption lines in the spectra. In the nuclei of two Seyferts, NGC 1068 and NGC 4051, there is some evidence for a stellar population like that of ordinary galactic nuclei (Andrillat and Souffrin, 1971). Possibly the highest stellar density observed in a Seyfert nucleus comes from the Stratoscope II photographs of NGC 4151. Assuming that most of the mass of its nucleus is in stars of $\sim 0.2 M_{\odot}(M / L \approx 20)$ which occupy about the same region as the non-thermal radiation, the properties of this nucleus are approximately (Schwarzschild, 1973) mass = $=4 \times 10^{9} M_{\odot}, L_{\text {vis }}$ (nonthermal) $=6 \times 10^{9} L_{\odot}, L_{\text {vis }}$ (stars) $=2 \times 10^{8} L_{\odot}$, number of stars $=2 \times 10^{10}$, diameter of nucleus $<7 \mathrm{pc}$, velocity of stars $>1500 \mathrm{~km} \mathrm{~s}^{-1}$, star collision rate $>2$ per year.

Since I have recently reviewed the properties and dynamical evolution of dense stellar systems elsewhere (Saslaw, 1973), they will be discussed only very briefly here. I shall try to sketch, in a very schematic way, the highlights of the evolution of a dense stellar system, but the previous review should be consulted for details, caveats, and references.

Dense stellar systems may form in a variety of ways. It could be that the initial conditions are very favourable. For example, in a cosmology with local inhomogeneities of large amplitude (represented by turbulence, lagging cores, great density perturbations, etc.) dense agglomerations may grow after matter and radiation decouple in the 
standard big-bang picture. These could then form the nuclei of galaxies, accreting the rest of the galaxy from initially more uniform surroundings.

But what if the relevant initial conditions (as determined by their other observational implications, if any) do not turn out to be so favourable? Suppose initial perturbations first grow into a gaseous, rather more uniform cloud. Such a cloud will normally have a density which decreases outward from its centre. Characteristically, in contracting, the central density rises more rapidly than the density in the outer parts, and the inhomogeneity becomes exacerbated. This situation is probably unstable, and the centre splits into stars (Larson, 1969).

Other processes can further increase the density of the nucleus. As stars lose mass during their natural evolution, some of the lost mass may devolve to the centre and there form new stars which in turn lose mass which falls further to the centre and there forms new stars which in turn... As an illustration (Spitzer, 1971), if 10\% of the entire stellar mass becomes gas which falls to the centre and forms new stars in a region with radius $5 \%$ of the original system, there is a density enhancement of $\sim 10^{3}$. Starting with $10^{11} M_{\odot}$ in a $10 \mathrm{kpc}$ radius, three such steps create a core with $10^{8} M_{\odot}$ in $1 \mathrm{pc}$. A system of stars with the solar luminosity function would evolve about $20 \%$ of its stellar mass into gas (of which about half is assumed to fall to the centre) after $\sim 10^{8} \mathrm{yr}$. Thus a dense core could be formed rather quickly in the evolution of a galaxy. This process depends on the gas having little or no angular momentum, and on fairly normal stars being formed at each stage.

Purely stellar dynamical effects will also tend to produce a dense core. If the system is nearly in equilibrium, the stars will perturb one another's orbits slightly. Some stars will slowly gain energy until they escape, and form a halo of high energy stars. Since the remaining stars have lower average energy (i.e. their total energy is more negative), they form a more compact core. The time scale for stars to evaporate is about

where

$$
T_{\mathrm{evap}} \approx 10^{2} T_{\mathrm{R}}
$$

$$
T_{\mathrm{R}}=8.3 \times 10^{5} \frac{N^{1 / 2} R_{(\mathrm{pc})}^{3 / 2}}{\left(M / M_{\odot}\right)^{1 / 2}(\log N-0.3)}{ }^{\mathrm{yr}}
$$

is the relaxation time for stellar orbits to be deflected substantially from their original motion. $R$ is the rms radius of the system having $N$ stars each of mass $M$. The core which remains behind becomes highly condensed after about 20 initial relaxation times $T_{\mathrm{R}}$. The evaporation time scale can be quite long. For example, use of the values for NGC 4151 given earlier with $R<7 \mathrm{pc}$, gives $T_{\text {evap }} \lesssim 4 \times 10^{13} \mathrm{yr}$. Unless this nucleus is much smaller than $7 \mathrm{pc}$, evaporation of stars will be negligible.

Formation of a dense core may be faster if the system relaxes violently. To do this it must start far from equilibrium. Then stars will scatter not just against small fluctuations in the mean gravitational field, but against large collective oscillations of the whole stellar system. If the gravitational field is inhomogeneous in space and varies rapidly in time, the time needed for relaxation may be decreased compared to $T_{\mathrm{R}}$ by a factor of as much as $N / \log N$, for the limiting case when the whole system oscillates 
with period $\sim(G \varrho)^{-1 / 2}$. Violent relaxation is quickly damped, however, by phase mixing and Landau damping, and the net results of this relaxation in a realistic system are not yet well understood.

Another effect which produces dense cores is the lack of energy equipartition among stars of different masses. If there were equipartition, the massive stars with lower velocities would sink to the centre of the system, and there form a quasi-stationary subsystem. Stars would evaporate so slowly compared to the crossing time (which measures collective gravitational response) that the system could still be considered to satisfy a quasi-stellar virial equilibrium. However, in nearly all systems there can be no equipartition. As a result, the more massive stars will lose kinetic energy to the lighter ones, fall toward the centre, continue to lose the kinetic energy they gain by falling, and continue to fall. The tendency of energy exchange to produce equipartition never succeeds, and ultimately a dense core forms at the centre. Although the dynamical evolution is not fully understood, numerical experiments suggest that the lack of equipartition may produce a core about an order of magnitude faster than in a comparable system with equal mass stars.

When the core becomes sufficiently dense, stars will collide bodily. For a typical star, the mean time between collisions is

$$
t_{\mathrm{c}}={ }_{n \sigma v}^{1}=\frac{9.7 \times 10^{21} R_{(\mathrm{pc})}^{7 / 2}}{N^{3 / 2}\left(m^{1 / 2} r^{2} / M_{\odot} r_{\odot}^{2}\right)\left(1+8.8 \times 10^{7} R_{(\mathrm{pc})} r_{\odot} / N r\right)}
$$

where $r$ is the radius of the star. In deriving this relation, the virial theorem has been used and the geometrical cross-section has been increased by a factor $\left(1+2 \mathrm{Gm} / \mathrm{r} V^{2}\right)$ to account for the gravitational attraction, ignoring tidal deformation. As examples, for $10^{8}$ stars and $R_{(\mathrm{pc})}=(0.1,1,10,100)$, we have $T_{\mathrm{R}(\mathrm{yr})}=\left(3.4 \times 10^{7}, 1.1 \times 10^{9}, 3.4 \times 10^{10}\right.$, $\left.1.1 \times 10^{12}\right)$ and $t_{\mathrm{c}(\mathrm{yr})}=\left(2.8 \times 10^{6}, 5.2 \times 10^{9}, 3.1 \times 10^{12}, 1.1 \times 10^{15}\right)$.

If two similar stars collide at relative velocities exceeding several hundred kilometres per second, most of the gas will interact supersonically relative to the local sound speed, and shocks will convert much of the kinetic energy of stellar motion into thermal energy which is then radiated. Thus the collision is highly inelastic. If, moreover, the total binding energy of the two stars substantially exceeds the total kinetic energy of their orbital motion, most of the stars' material will coalesce. The distended, newly formed object pulsates for some time, then settles down as a well-defined star. Eventually it may become a supernova.

If the velocities of the stars are so great that their orbital kinetic energy exceeds their gravitational energy, the collision will be mostly disruptive. For two sun-like stars this implies a relative velocity $V \gtrsim 1500 \mathrm{~km} \mathrm{~s}^{-1}$, approximately. Using simple models, a number of calculations of colliding stars were made some years ago (Spitzer and Saslaw, 1966; Mathis, 1967; Colgate, 1967; De Young, 1968; Sanders, 1970; Seidle and Cameron, 1972). These have greatly clarified, but not really solved, many basic questions such as the amount of mass loss, the conditions for coalescence, the growth of very massive stars, the role of thermonuclear reactions, the generation of relativistic 
particles. Other questions such as the structure and evolution of a coalesced star, the most massive stars that can form by coalescence, and the fraction of stars in the nucleus that survive the coalescence phase are still quite open.

Geometric collisions begin to dominate the evolution of the core when the collision time $t_{\mathrm{c}}$ becomes less than $T_{\mathrm{R}}$. This happens when $R \approx N r$, so that if the whole system were put into one dimension with the stars just touching one another, the length of this line would be about the radius of the three-dimensional system. Initially most collisions occur at low velocities and the stars coalesce. At first, the main sequence lifetime of the coalesced stars is less than the collision time, so the newly combined stars have a chance to evolve off their main sequence, perhaps becoming supernova. Collisions are not yet so rapid that they add fuel to stars and mix their cores faster than the hydrogen is burned. The general evolution of a cluster during this period has been simulated by Monte Carlo calculations (Sanders, 1970). Although the results are quite model-dependent, it is easy to find plausible conditions which produce the approximate luminosities and lifetimes of Seyfert galaxies and quasars.

As the system contracts still further, coalescing collisions become more frequent until the collision time becomes less than the time for the coalesced star to evolve into a supernova. If enough time is spent in this regime (before disruptive collisions take over), stars of extreme mass may form. At first the mass of a typical star is built up by coalescence with smaller stars. Every addition of hydrogen with mixing is assumed to be so effective that it sets the star's evolutionary clock back to zero (an important question for further calculation). In this way stars of $\sim 500 M_{\odot}$ may form (Sanders, 1970). These in turn sink to the centre and, coalescing one with another, accelerate the building of even more massive stars. Eventually the cluster becomes so compact and the velocities so high that it enters a phase dominated by the disruption of the objects which have already grown.

In systems where a large number of low mass stars survive the coalescence phase (what detailed conditions are necessary for this?), the subsequent evolution will be even more complex and dramatic. Collisions at velocities $\gtrsim 2000 \mathrm{~km} \mathrm{~s}^{-1}$ liberate appreciable amounts of gas from the stars. This gas cools by free-free emission and radiation by heavy ions, especially carbon, nitrogen and oxygen. A simple homogeneous model (Spitzer and Saslaw, 1966) suggests that when enough gas is liberated, its cooling time can become substantially less than the collision time of the stars. As the temperature falls the cloud begins to shrink, its density rises, and the cooling processes accelerate rapidly. As a result, the pressure gradient no longer supports the gas, and the cloud goes into free-fall. Beginning with negligible interstellar matter, this sequence of the building, cooling, contraction, and collapse of a gas cloud takes $\sim 5000 \mathrm{yr}$ and involves $\sim 200 \mathrm{M}_{\odot}$ in a nucleus containing $10^{9}$ stars within a $1 \mathrm{pc}$ radius (about $5 \times 10^{3}$ ions $\mathrm{cm}^{3}$ ). It takes $\sim 200 \mathrm{yr}$ and involves $\sim 4000 \mathrm{M}_{\odot}$ for a nucleus with $10^{10}$ stars in $1 \mathrm{pc}$ and it takes $\sim 2 \times 10^{7} \mathrm{yr}$ and involves $\sim 50 \mathrm{M}_{\odot}$ for $10^{9}$ stars in $10 \mathrm{pc}$. At this stage of evolution the cooling of the gas is so rapid that the average mass of gas in the nucleus is too small to influence its dynamics, except at the very centre.

The gas which falls to the centre forms a flattened disk whose radius is determined by 
its angular momentum. Since stars with opposite orbital angular momentum have a somewhat greater probability of colliding than stars moving in the same direction, it is likely that the specific angular momentum of the liberated gas will be less than that of the stars. The rms height of the gas above the disk, on the assumption of hydrostatic equilibrium, is approximately (Spitzer, 1942) $Z_{\mathrm{g}} \approx R V_{\text {gas }} / V_{\text {stars }}$, where $R$ is the rms radius of the stellar system, $V_{\text {gas }}$ and $V_{\text {stars }}$ are the rms velocities of these components of the system. Since $V_{\text {stars }}$ must be at least $\sim 2000 \mathrm{~km} \mathrm{~s}^{-1}$, and the gas may cool below $10^{4} \mathrm{~K}\left(V_{\text {gas }} \lesssim 10 \mathrm{~km} \mathrm{~s}^{-1}\right)$, the disk is very thin compared to the stellar system.

At this stage (and neglecting the more exotic possibilities to be discussed later), there are four main sources of luminosity in the nucleus: the general background of old stars including coalesced objects, the radiation of gas liberated by collisions during the collision and later as it falls toward the centre, new $\mathrm{O}$ stars or supermassive stars formed in the disk, and supernovae. If we assume that an energy $\frac{1}{2} V_{\text {stars }}^{2}$ per unit mass of gas liberated is radiated as the gas cools, and an equal amount is lost as the gas falls into the disk radiating the potential energy released, then the luminosity of the gas $L \approx V_{\text {stars }}^{2} \xi M / 2 t_{\mathrm{c}}$. Here $\xi$ is the fraction of the total mass lost per collision. Substituting the virial expression for $V_{\text {stars }}$, and the previous equation for $t_{\mathrm{c}}$, gives

$$
L \approx 7 \times 10^{10_{\zeta}} N^{7 / 2}\left(m / M_{\odot}\right)^{5 / 2}\left(r / r_{\odot}\right)^{2} R_{(\mathrm{pc})}^{-9 / 2} \mathrm{erg} \mathrm{s}^{-1}
$$

For velocities of $1500-2000 \mathrm{~km} \mathrm{~s}^{-1}, \xi \approx 0.06$ and, as an illustration, if there are $10^{8}$ suns in $\sim 0.05 \mathrm{pc}$ radius, then $L \approx 10^{43} \mathrm{erg} \mathrm{s}^{-1}$. The contribution of the other three sources could exceed this luminosity, depending on details of the model.

The late evolution of the central disk depends strongly on its gravitational interaction with the rest of the nucleus. If the gas forms stars of fairly normal mass (which is by no means guaranteed), the degree of mixing between old and new stars is governed by the ratio of the relaxation time, $T_{\mathrm{R}}$, to the timescale $t_{\mathrm{c}} / \xi$ for collisions to liberate a substantial fraction of the entire stellar mass. As long as this ratio remains small, distant encounters between the low-velocity new stars with the old high-velocity ones are able to transfer enough kinetic energy to enable the newly formed stars to rise from the disk. At the same time, the orbits of old stars contract further and the average density increases. It is difficult to determine the actual amount of energy exchange, and models have been constructed (Spitzer and Stone, 1967) for the two extremes of zero exchange and of the maximum exchange possible. The luminosity of the liberated gas depends strongly on the angular momentum of the gaseous disk, and is greatest in cases of low angular momentum. Plausible models give luminosities and lifetimes characteristic of Seyfert nuclei and quasars during this phase.

When the core becomes so tight that $\xi T_{\mathrm{R}} / t_{\mathrm{c}}>1$, collisions destroy most of the older stars before they have time to impart their kinetic energy to the younger ones. Gradually the stellar system becomes a flattened disk, the random stellar velocities are greatly reduced, and the luminosity produced by the colliding stars begins to die away. But even this state does not end the career of the nucleus. 
Now, if not before, a new menagerie of objects may grow to affect the further evolution of the nucleus. These are the more speculative members of the second list. But before describing their roles, let us pause to face the basic question: Is there one main source of energy in the nucleus, or are there many?

Evidence on this question is scanty, and comes mainly from the nature of intensity variations in the objects. The longest and most detailed optical variations are for the quasar $3 \mathrm{C} 273$, for which there is some suggestion of a $\sim 10 \mathrm{yr}$ cycle. This has given rise to an inconclusive quasi-periodic debate in the literature as to whether the light curve can be represented by a superposition of randomly occurring outbursts. For the latest instalment, with references to previous debate, see Chertoprud et al. (1973). In the radio, the most extensively observed variations are for the Seyfert-like galaxy 3C 120 (Dent, 1972a) and for the quasar 3C 279 (Dent, 1972b). Both these objects vary substantially on a time scale of weeks. If they are coherent objects, they give the impression of expanding faster than the speed of light. The alternative explanations of this faster-than-light expansion are that the objects are really much closer than the Hubble relation applied to their redshift indicates, that the expansion is a phase velocity effect, or that short-lived outbursts occur fairly randomly in various parts of the object. Very few astronomers at this time feel that the evidence for the breakdown of the Hubble law is compelling. The second explanation requires rather artfully contrived, but still possible, models. The third explanation seems to be the simplest. A possible problem with this random event model - sometimes likened to lights blinking on and off on a Christmas tree - is that in some cases successive brightenings tend to occur along the same line on the sky. This would require that we see a disk nearly edge-on. However, there are not yet enough of these objects to determine whether the required geometry is statistically improbable.

In addition to evidence from individual objects, there is some information for the statistical properties of radio spectra in compact extragalactic objects (De Young, 1971). Nearly 50 sources, including both quasars and radiogalaxies, have more than one intensity peak in their spectrum. If these peaks are due to synchrotron self-absorption of radiation from an outburst, then the size and magnetic field strength of the outburst can be found from the frequency and flux density at the peak, if an average brightness temperature (which is not very critical) is assumed. The distribution of these peaks seems to be more consistent with multiple outbursts occurring in physically separate regions than with successive outbursts from a central source into a preexisting or regenerated magnetic field.

Although the balance of evidence seems to favour the multiple source model, it is not at all decisive. There is no reason why both types of source could not be present in the same object. The discovery of a galaxy or quasar with unambiguous periodic fluctuations would go a long way to showing that this could be the case.

With this background, we consider the forms that single sources might take. In olden days, cartographers drawing maps of unexplored regions of space often populated them with myriads of strange phantastical creatures. So also today, at the edge of understanding, we try to imagine the denizens of galactic nuclei. 


\subsection{SUPERMASSIVE STARS AND DISKS}

Historically, hot supermassive stars (Hoyle and Fowler, 1963a, 1963b) were the first single sources proposed for the energy of strong radio sources and quasars. If these objects of $\sim 10^{5}-10^{10} M_{\odot}$ were stable, they could burn thermonuclear energy with a luminosity of $\sim 10^{45} \mathrm{erg} \mathrm{s}^{-1}$ for $\sim 10^{6} \mathrm{yr}$. However, it has become apparent that non-magnetic, non-turbulent, non-rotating supermassive stars are difficult to form and stabilize, and recently this has been computed in detail (Appenzeller and Fricke, 1972; Fricke, 1973). As a supermassive star forms (either from a gas cloud or from coalescence of less massive stars) and begins to contract, there are four possible ways it may develop. If $M \lesssim 4 \times 10^{5} M_{\odot}$, it settles down into thermonuclear equilibrium. But if $M \gtrsim 4 \times 10^{5} M_{\odot}$, it can explode or collapse into a black hole. Explosion occurs, for a given mass, if the initial heavy element is great enough to produce rapid thermonuclear burning. For example, if $M=10^{6} M_{\odot}$, explosion occurs if $z>0.04$. When $z$ is too small for a given $M$, or $M$ is too great for a given $z$, thermonuclear energy cannot halt the gravitational collapse. The fourth possibility is that relaxation oscillations occur in which the radius, luminosity, and rate of energy production change periodically in the pulsating star. But this does not seem to occur unless the rate of burning is arbitrarily damped during the explosive phase.

It is worth noting that thermal radiation from the surface of stable supermassive stars will not generally have a Planck spectrum (Illarionov and Sunyaev, 1972). When electron scattering is more important than free-free processes in the outer atmosphere, the radiation spectrum can be quite complex, depending on the run of temperature and density in the atmosphere. For a simple power-law dependence of plasma temperature on surface depth, the radiation may follow a power-law spectrum with negative index, thereby mimicking the spectrum of synchrotron radiation over a range of frequencies.

To overcome some of the stability problems of supermassive stars, one can consider differentially rotating supermassive disks (e.g. Salpeter and Wagoner, 1971; Quirk and McKee, 1971; Scharlemann and Wagoner, 1972). Unlike stars in which gravity is opposed by thermal gas and radiation pressure, these disks are maintained by the balance of gravitational and centrifugal forces. If the matter is completely cold, the disk will be infinitesimally thin. In the more realistic case where the pressure, total energy density, and redshift at the centre of the disk are $P_{\mathrm{c}}, E_{\mathrm{c}}$, and $z_{\mathrm{c}}$ respectively, the ratio of the half-width to the equatorial radius is approximately (Salpeter and Wagoner, 1971)

$$
\frac{W}{R} \approx \frac{P_{\mathrm{c}}}{E_{\mathrm{c}}} \frac{1+z_{\mathrm{c}}}{z_{\mathrm{c}}},
$$

for an idealized uniformly rotating disk, and the period of rotation of the disk is approximately

$$
\tau \approx 8 \times 10^{-5}\left(M / M_{\odot}\right)\left(\begin{array}{c}
c J \\
G M^{2}
\end{array}\right)^{3} \mathrm{~s},
$$


where $J$ is the angular momentum. To within a factor of order unity, $z_{c} /\left(1+z_{c}\right)$ is the ratio of the maximum binding energy to the rest mass energy. For a hot disk,

$$
\frac{L}{L_{\odot}} \approx 3 \times 10^{4} M / M_{\odot} .
$$

An important difference between disks and stars is that when a pressure-supported star loses only angular momentum, its radius does not change significantly and therefore its rotation slows down. But a centrifugally-supported disk contracts and rotates faster when it loses angular momentum. The disk cannot rotate too rapidly, or it will begin shedding mass. And if it rotates too slowly, it collapses. The exact regime of stability depends on the detailed rotation curve.

Several results on the stability of these disks are described by Quirk and McKee (1971). For a maximum global stability against collapse, and local stability against fragmentation, one wants a rotating, very centrally condensed, disk supported either by radiation pressure or a tangled magnetic field (particle pressure does not give sufficient internal energy for stabilization). The minimum stable ratio of thickness to radius for a uniformly rotating Newtonian disk is about $1 / 20$; differential rotation can reduce this by a factor of $\sim 2$, but relativistic effects may increase it by a factor of 1-2 for $R / R_{\text {Schwarzschild }} \lesssim \frac{1}{2}$. These results are based on analytic idealizations, and numerical experiments suggest they may be off by about $50 \%$, to give some idea of the uncertainty in these stability calculations. No realistic stability calculations have yet been done for highly-relativistic, differentially-rotating disks supported by magnetic pressure.

For $R / R_{\mathrm{Sch}} \approx \frac{1}{2}$, the disk can release $\sim 6 \%$ of its rest mass energy during contraction to this radius, and possibly more if it accretes surrounding gas and dust. Disks supported by radiation pressure radiate thermally at the rate (Quirk and McKee, 1971)

$$
L \approx 1.3 \times 10^{38} \frac{M}{M_{\odot}}\left(1-R / R_{\mathrm{Sch}}\right) \mathrm{erg} \mathrm{s}^{-1} .
$$

Therefore the time for them to lose all their pressure support and become infinitesimally thin, if no processes other than thermal radiation are involved, is

$$
E_{\text {binding }} / L \approx 10^{8} \underset{R}{R_{\mathrm{Sch}}}\left(1-\frac{R_{\mathrm{Sch}}}{R}\right)^{-1} \mathrm{yr} .
$$

But with only thermal radiation losses the disk would contract to an unstable axial ratio of $\sim \frac{1}{20}$ in about $3 \times 10^{7} R / R_{\text {Sch }}$ yr. So if the disk is to avoid fragmenting, it must contract even faster, which means that non-thermal energy losses (e.g. magnetic dipole radiation) must be more important than thermal losses.

Fragmentation is not a particularly great evil, however; it is just difficult to understand in detail. If the disk fragments, its early evolution will depend on the mass and size of the fragments relative to the central condensation in the disk. If the outer part of the disk (which may be more unstable if its differential rotation is less) 
fragments into many small objects, these might orbit the centre as a reasonably stable satellite system, perhaps surrounding a black hole. On the other hand, if a few massive fragments form, the system would probably be quickly unstable: some pieces might be ejected and others would coalesce as they radiated gravitational waves. The effects of tidal forces make the picture more uncertain. No one knows whether the fragments would be neutron stars, smaller disks, or black holes. We should also remember that the instability may be set off not by an infinitesimal perturbation, but by a collision of the disk with a massive star or supernova, or by the eruption of a magnetic flare, and this could change the nature and growth rate of fragments considerably. These problems are very difficult, and our lack of understanding of Newtonian fragmentation and star formation just increases the uncertainties (cf. Arny and Weissman, 1973). Moreover, among all these questions lurks the possibility that the equations of general relativity are not applicable to strong gravitational fields - where they have never been tested.

\subsection{RotATING MAGNETOIDS}

In many massive rotating objects, a magnetic field may be important. These configurations have been called magnetic rotators, magnetoids, or spinars. They come in two main forms: hot and cold. Hot magnetoids, with high entropy per baryon, are the more spherical in shape and are supported by radiation pressure as well as by the magnetic field. There are a variety of possible structures, depending upon whether the rotation is uniform or differential, and upon the poloidal and toroidal components of the magnetic field (Ozernoy and Usov, 1971). A poloidal field, however, will tend to smooth differential rotation in a time short compared to the evolutionary contraction time of the magnetoid. To account for the activity in galactic nuclei (and radiogalaxies) these objects must have a lifetime of $\gtrsim 10^{5} \mathrm{yr}$. There are two kinds of hot magnetoids which seem to have this property. Either they have differential rotation and a very weak poloidal field, or they rotate uniformly and have a strong poloidal field. In the first case, however, the field is too small to give appreciable magnetic dipole radiation compared with thermal radiation, so this configuration is unstable to fragmentation.

The thermal luminosity of hot magnetoids is determined approximately by the Eddington limit at which radiation pressure balances gravity:

$$
L_{\text {thermal }} \approx 10^{46}\left(M / 10^{8} M_{\odot}\right) \operatorname{erg~s}^{-1},
$$

although, as mentioned previously, the emerging Planck spectrum could be substantially modified by electron scattering. The magnetic dipole radiation has been considered by Cavaliere et al. (1971) and in more detail by Ozernoy and Usov (1973a, b). It is appreciable for at least $\sim 10^{5} \mathrm{yr}$ in a uniformly rotating object with a strong poloidal field. At the poles, this magnetic field has the value

$$
B_{\mathrm{p}} \approx 10^{6} \xi^{1 / 2}\left(M / 10^{8} M_{\odot}\right)\left(R / 10^{16} \mathrm{~cm}\right)^{-2} \mathrm{G}
$$

where $\xi$ is now the absolute value of the ratio of total magnetic energy to gravitational 
energy. If the rotation is as fast as it can be without disrupting the star, i.e. $\Omega \approx\left(G M / R^{3}\right)^{1 / 2}$, this field gives the luminosity of magnetic dipole radiation as

$$
L_{\mathrm{md}} \approx 10^{58} \xi \sin ^{2} \chi\left(R_{\mathrm{Sch}} / R\right)^{4} \mathrm{erg} \mathrm{s}^{-1},
$$

where $\chi$ is the angle between magnetic and rotational axis. This angle may change in a quite complicated manner which depends on how angular momentum is removed from the object. In particular, the rate of loss of angular momentum depends sensitively on the temperature of the object's corona (Anand and Shara, 1972).

The spectrum of this luminosity has not been worked out rigorously for a realistic situation. But the general idea is that intense low frequency $\left(v \approx 10^{-6}-10^{-8} \mathrm{~Hz}\right.$ for very massive rotators) radiation accelerates the surrounding plasma to relativistic velocities. These high energy particles then radiate both by synchrotron radiation with the dipole field, and by Compton scattering off the low frequency waves which accelerated them (Blandford and Rees, 1972). Simple models suggest that this is a plausible mechanism for producing radio and infrared emission.

As the hot magnetoids radiate, they cool and any remaining support must come from rotation and an internal magnetic field. Thus they flatten and come to resemble the disks of the previous section. The structure of a thin, magnetic, uniformly-rotating disk has been determined by Scharlemann and Wagoner (1972), but little is about known its detailed stability.

Perhaps the most unequivocal observational test for these objects would be the discovery of a definite dominant periodicity in the luminosity of a quasar or active galaxy. So far, this does not seem to have been found, and the other evidence that they exist in quasars is not compelling (Sturrock, 1971). However, they cannot be ruled out.

\subsection{Pulsars}

Since pulsars can produce moderate amounts of radio and optical radiation, it seems attractive to gather $\sim 10^{7}$ of them together to explain the activity in galactic nuclei and quasars (Kardashev, 1970; Rees, 1971). The main problem is to account for the 1-100 supernovae per year over $\sim 10^{7} \mathrm{yr}$ in the nucleus needed to produce these pulsars (if supernovae make them with high efficiency). Pressent models for supernova generation during the formation of galaxies are phenomenological, so one simply assumes the parameters required to fit the observations. (Un)fortunately for these models, there are always more parameters than observations. The most detailed model of galactic nuclei as pulsar clusters is by Arons et al. (1974). In particular, they consider collective effects such as the acceleration of particles scattered by moving pulsars in analogy to the Fermi mechanism, and the efficient resonant acceleration of particles by the superposed wave fields of the pulsars, especially when two pulsars have the same frequency in the Doppler-shifted particle frame. These models can be made to agree reasonably well with the observed luminosities, fluctuations, and optical lines. However, their necessary complexity inhibits any definitive tests. 


\subsection{SingULARITIES IN GENERAL RELATIVITY}

When the ratio, $G M / r c^{2}$, of the gravitational binding energy of an uncharged, nonrotating object to its rest mass energy becomes as large as unity, an event horizon forms around the object and prevents radiation from escaping from the Schwarzschild radius $R_{\mathrm{Sch}}=2 G M / c^{2}$. For rotating or charged objects this criterion is modified somewhat, but still a black hole forms. It is an unsolved question as to whether all types of objects have event horizons when their self-gravitational field is sufficiently strong, or whether there can exist 'naked singularities' without such an horizon.

How could we tell whether there is a black hole in the nucleus of a galaxy? As the hole forms, it is unlikely to retain any evidence of a magnetic field if the net charge is zero (Scharlemann and Wagoner, 1972). Thus the black hole itself probably doesn't produce any electromagnetic radiation, although surrounding gas flowing into the hole may drag a magnetic field with it and radiate. The possibility of detecting a black hole when it accretes will be discussed briefly in the next section.

A black hole might be detected, in principle, by its modification of the light from a surrounding cloud of stars. Gerlach (1971) has examined the idealized case of a collisionless system of stars moving in a spherical shell around a black hole. Each star radiates at the same frequency in its own rest frame, and the photons move through the Schwarzschild geometry without scattering or absorption, but the random Doppler motions of the stars broaden the spectrum. The effect of the black hole is to swallow photons emitted toward it with impact distances less than $\sqrt{27} R_{\mathrm{Sch}} / 2$. This first of all depletes the redshifted photons (as would be seen by a distant observer) relative to blueshifted photons, below what the flux would be without the black hole. The amount of depletion depends mainly on the distance of the stars from the hole, but even for the closest stable orbit it is not more than $40 \%$. Secondly, the total photon intensity, integrated over all frequencies, is also depleted by about $50 \%$ (relative to the case of no black hole) at these small impact distances. The system would appear to be a faint central disk surrounded by a ring whose inner edge is about twice as bright as the disk, and whose outer edge is about three times as bright. Unfortunately, to observe even the largest black hole $\left(\sim 10^{11} M_{\odot}\right)$ in the nearest large galaxy (M31) in this way would require a resolution $\lesssim 10^{-2}$ arc sec. Moreover, a realistic distribution of stars, and any gas or dust present in the nucleus, will probably smooth this picture beyond recognition.

The gravitational effect of a black hole on the stellar distribution in the nucleus might be detectable under extreme conditions, but the details of this effect are uncertain. If one assumes that the stellar distribution around a black hole reaches a steady state in which the distribution function, $f(r, v) \propto E^{\mathrm{p}}$, is a power of the total energy $E=(G M / r)-\frac{1}{2} v^{2}$, then the star density $\varrho \sim r^{-9 / 4}$ and the velocity dispersion $v \sim r^{-1 / 2}$ (Peebles, 1972) where $r$ is radial distance from the hole. To within observational uncertainties this density distribution cannot be distinguished from an isothermal sphere, $\varrho \sim r^{-2}$. The theoretical uncertainties in the form of $f(r, v)$ are probably at least as large, especially if the stars have a range of masses and do not reach a steady state. 
Wolfe and Burbidge (1970) discuss in some detail the possibility that galactic nuclei contain black holes. They also assume that the stars in the nucleus are relaxed but in the form of an isothermal sphere, which is at least quasi-stationary. This is consistent with observations showing a smooth luminosity distribution into the innermost $1^{\prime \prime}$ radius, at which point the seeing disk smears the image. If the presence of a black hole at the centre is not to change the isothermal nature of the stellar distribution significantly (a problem deserving more study), then a simple model gives upper limits to the possible mass of a central black hole from observations of the luminosity and velocity distributions. For most giant elliptical galaxies, this upper limit is $\sim 10^{10} M_{\odot}$. A central black hole cannot, therefore, explain the high visual mass/luminosity ratios of these galaxies, but it could account for explosive phenomena in some nuclei. It is possible, however, for there to be a large number of holes, with the same mass spectrum as the stars, spatially distributed like stars throughout these galaxies.

Another point of view is to regard the very existence of galaxies as evidence for black holes in their nuclei (Ryan, 1972). As a way out of the well-known problems of forming galaxies from statistical fluctuations in an initially homogeneous universe, one can start with a finite amplitude perturbation after matter and radiation decouple, and have the perturbation accrete a galaxy (never mind the origin of this perturbation itself, according to this view). This idea was first suggested in the context of steadystate cosmology (Roxburgh and Saffman, 1965), but is more broadly applicable. A perturbation of $\sim 1 \%$ the mass of the final galaxy works very well in most cosmological models, but there is no real necessity for it to be a black hole.

\subsection{ACCRETING OBJECTS}

This category is not concerned so much with a particular type of object, as with ways in which objects may swallow their surroundings. The objects may be black holes, neutron stars, or relativistic disks. The basic physics of accretion is reviewed in Zeldovich and Novikov's (1971) book, and more recently in Novikov and Thorne (1972), so I shall make very few comments here. The subject has been revived in recent years, mainly as a possible explanation for X-ray stars.

Radiation produced by accreted matter ultimately comes from the gravitational potential energy of the objects it falls onto. This energy is greatest in the case of a black hole, even though it is redshifted substantially as it escapes from regions close to the event horizon (the Schwarzschild radius in the case of an uncharged, non-rotating hole). Orbits of stars and other point masses moving around a Schwarzschild singularity are unstable if (Hansen, 1971) $f \leqslant(3+e) R_{\text {Sch }}$ where $f$ is the semi-latus rectum and $e$ is the eccentricity. Since real stars have a finite size, they will be broken up by tidal forces on reaching the unstable orbits. Viscous dissipation (possibly magnetic) causes the resulting gas and dust to radiate and fall into the unstable region where it is swallowed for ever. The manner in which stars surrounding a black hole interact to populate the unstable orbits is an important problem on which Wolfe and Burbidge (1970) have made a start. The motion of gas and stars into rotating Kerr black holes is a more involved problem which is discussed by Wilson (1972), and Bardeen et al. (1972). 
A fundamental question is how much of the energy of a black hole can be extracted under astrophysically plausible conditions. In principle $\sim 42 \%$ of the accreted restmass energy could be radiated in a Kerr metric (Bardeen, 1970), but this may not be realized in practice. Even for spherically symmetric, non-magnetic, steady-state accretion of pure hydrogen onto a Schwarzschild black hole, the luminosity and spectrum depend very sensitively on the mass of the hole, and the temperature and density of accreted gas (Shapiro, 1973). Models of accretion onto black holes (Lynden-Bell and Rees, 1971; Norman and ter Haar, 1973) in active galactic nuclei suggest that masses $\sim 10^{8} M_{\odot}$ are needed.

Accreting neutron stars may also serve as an energy source for galactic nuclei (Bisnovatni-Kogan and Sunyaev, 1972). As with pulsars, one must account for the high concentration of neutron stars in the galactic nucleus. Also, only the dying neutron stars which are not energetic pulsars would be able to accrete substantially. Recently much work has been done on the properties of neutron star accretion in connection with X-ray sources, but that is beyond the scope of this review.

\subsection{ANTI-MATter}

If the infrared radiation of Seyfert nuclei is produced by matter-anti-matter annihilation, the minimum flux of $\mu$-neutrinos which is also produced can be predicted for straightforward models (Steigman and Strittmatter, 1971). The original observations of high infrared flux were inconsistent with the low observed upper limits of the $\mu$-neutrino flux. More recent estimates which lower the IR flux are consistent. However, in view of the present difficulties with solar $e$-neutrino expectations, any conclusions about anti-matter are very tentative.

\subsection{Gravitational Radiation}

In galactic nuclei, gravitational radiation can be generated by collisions of stars or black holes, or during the accretion process itself. Very little of the radiation is absorbed by other matter; most escapes to transport energy and angular momentum out of the galaxy. This could remove much of the mass from galaxies. Recently Press and Thorne (1972) have reviewed the properties, production, and detection of gravitational waves.

\subsection{NEW PHYSICS}

There have been a number of proposals, starting with Jeans (1929) and including Ambartsumian (1965), that entirely new physics is needed to explain the violent activity in galactic nuclei. Ambartsumian, particularly, has advocated the view that the galaxies themselves form by the splitting and expansion of very condensed matter. Variants of this view consider lagging cores from the early big-bang Universe (Novikov, 1965; Ne'eman and Tauber, 1967; Harrison, 1971) or pockets of creation in the steady-state cosmology (Hoyle and Narlikar, 1966). This is in contrast to the more general view that galactic nuclei form from the contraction of regions of diffuse gas. A definitive observational test of whether galaxies form from the inside out or from the outside in 
would be a great step forward. So far, it seems that the features of galactic nuclei can be explained reasonably well by applying conventional physics (including general relativity), and there is no necessity to modify any basic laws. However, this question deserves an open mind.

\section{The Ejection of Matter from Galactic Nuclei}

Thus far we have mainly tried to learn about galactic nuclei by understanding their manner of emitting radiation. There is substantial evidence that many galaxies also eject large quantities of gas, and perhaps massive compact objects as well. The observations were reviewed by Burbidge (1970) and at the Vatican Symposium (O'Connell, 1971), as well as at this Symposium. In addition to all the evidence for the explosion of some galaxies (e.g. M82, NGC 1275) and the ejection of radio sources from many others, there is an increasing number of anomalies such as the compact components of the nucleus of NGC 1808 which may be dynamically unstable (Arp and Bertola, 1970), the blue condensations associated with elliptical and S0 galaxies (Stockton 1972), and the broad $\left(\sim 13000 \mathrm{~km} \mathrm{~s}^{-1}\right) \mathrm{H} \alpha$ lines in the Seyfert I 4329 A which may indicate high outflow velocities (Disney, 1973). Such phenomena may contain important clues to the inner workings of galactic nuclei.

What sets off these vast explosions? In each case, the ultimate source of the energy is gravitational, but the acceleration may be caused directly by magnetic fields, radiation pressure, or hydrodynamic explosions, as well as by gravity. Let us consider each of these possibilities in turn.

Magnetic sources of acceleration arise through the rotational winding up of magnetic fields until they become unstable. One process, numerically calculated by Le Blanc and Wilson for stars (see Wheeler, 1971), may also apply to more massive objects. They consider a collapsing, rotating star of $\sim 7 M_{\odot}$ and initial central density $\sim 10^{8} \mathrm{~g} \mathrm{~cm}^{-3}$. It starts with a small poloidal magnetic field $\left(E_{\text {magnetic }}=-2.5 \times\right.$ $\left.\times 10^{-4} E_{\text {grav }}, E_{\text {rot }}=-2.5 \times 10^{-3} E_{\text {grav }}\right)$ which is wound up by differential rotation as the star contracts. The field becomes mostly azimuthal and builds up great pressure along the axis of the star. In a very short time, when the central density reaches $\sim 10^{11} \mathrm{~g} \mathrm{~cm}^{-3}$, this field bursts out along the axis producing a double-sided jet. Although the material in the jet goes off with high velocity, $\sim 0.1 \mathrm{c}$, only $\sim 1.5 \times 10^{-3}$ of the star's mass is ejected, so the process is not very efficient. Whether it becomes more efficient in more massive stars is an open question.

Related models involve magnetic flares in galaxies and large differentially rotating gas clouds. Ozernoy and Somov (1971) consider such a contracting cloud, and are especially interested in the way rotation twists the initially poloidal magnetic field to form neutral lines and the field becomes quasi-radial. With continued twisting the field lines become unstable and a flare may occur, accelerating particles along the polar axis to relativistic velocities. This action would repeat as the cloud contracts further and rotation continues to tighten the residual field lines. Using a different geometry consisting of an annulus and a core rotating about the same axis but with different 
velocities, Sturrock and Barnes (1972) have indicated how a metastable force-free field can form. This may be explosively unstable and produce a double jet. Since these models are so complex, it is impossible to compute much MHD detail, but they make plausible the ejection of large clouds of relativistic gas approximately along the rotation axis.

Shklovsky (1970) has proposed, in general terms, a variant of magnetic ejection in which the relativistic particles and synchrotron radiation are ejected anisotropically from the magnetoid. Conservation of linear momentum implies that the magnetoid recoils and might be ejected from the galactic nucleus. The frequent similarity between double components on opposite sides of a radiogalaxy is not naturally explained by this approach, however, since one component would be a magnetoid and the other a cloud of gas.

Ejection driven by radiation pressure, and line-locking in particular, was discussed in Section 1.1. There may be some evidence that this occurs in the exploding Seyfert NGC 1275, alias 3C 84, alias Perseus A. Photographs and spectra (Burbidge and Burbidge, 1965; Lynds, 1970) of NGC 1275 show a central amorphous region with medusa-like filaments (rather like the Crab Nebula) coming out. The recession velocity of this region and its filaments is $5270 \mathrm{~km} \mathrm{~s}^{-1}$. On the northwest side of this central region there is a sector of $\sim 110^{\circ}$ in size containing a number of emission-line filaments with recession velocities of $8220 \mathrm{~km} \mathrm{~s}^{-1}$, or $+2950 \mathrm{~km} \mathrm{~s}^{-1}$ with respect to the systemic velocity. The remarkable thing is that these filaments extend throughout $\sim 10 \mathrm{kpc}$ from the centre (modulo unknown projection factors) and their velocities are all in the narrow range $8090-8372 \mathrm{~km} \mathrm{~s}^{-1}$, i.e. they cluster around the relative velocity of $\sim 3000 \mathrm{~km} \mathrm{~s}^{-1}$ to within $\pm 5 \%$. Dividing the distance of the farthest filament from the nucleus by $3000 \mathrm{~km} \mathrm{~s}^{-1}$ gives an age of $\sim 5 \times 10^{6} \mathrm{yr}$. It seems that ejection at the same velocity is still going on at the present time (De Young et al., 1974). Within $10 \mathrm{pc}$ from the nucleus is a cloud of neutral hydrogen, seen at $21 \mathrm{~cm}$ absorbed against one of the complex central radio sources, moving outward from the nucleus at $2850 \mathrm{~km} \mathrm{~s}^{-1}$. The width of the $21-\mathrm{cm}$ line is only $\sim 10 \mathrm{~km} \mathrm{~s}^{-1}$. Thus the cloud has a very low temperature and little turbulent motion.

There is no unique explanation of this cloud. For example, it could be a chance coincidence in position and velocity space, or it could be a small colliding galaxy. If these explanations don't apply, then the only method people have suggested which might accelerate gas to a very narrow velocity range for a long period of time is linelocking. Explosions or galactic winds won't do because they have too many parameters, and not enough known constraints. Now the second and third methods of line-locking discussed in Section 1.1., i.e. between two absorption lines, or between the Lyman continuum drop and an absorption line to its red are likely to the most persistent over long times. For the second possibility, one could consider strong Ly $\alpha$ absorption at $1215 \AA$ and the strong $\mathrm{N} v$ multiplet at $1238 \AA$. The velocity difference of these two absorption lines is $\sim 6000 \mathrm{~km} \mathrm{~s}^{-1}$, giving a velocity projection factor of $\sim 2$. Alternatively, one might consider the difference between the Lyman continuum drop and absorption multiplets of O IV $(922 \AA)$ and $\mathrm{N}$ IV $(923 \AA)$, giving a velocity of $\sim 3000$ 
$\mathrm{km} \mathrm{s}^{-1}$, and a projection factor $\sim 1$. However this latter possibility has the problem that the $\mathrm{O}$ IV and $\mathrm{N}$ IV lines are not resonance lines, and are therefore less likely a priori to give strong absorption. Thus it is possible that a line-locking mechanism is working. On the other hand, the combined hydrodynamic and radiative transfer problems have never been calculated, and it is not clear that this method will accelerate clouds coherently. Clearly, exciting things are happening here, and they are close enough to study in detail.

Moving toward hydrodynamic theories of ejection, we first encounter the buoyant bubble model (Gull and Northover, 1973). In this picture, relativistic particles in the nucleus are supposed to form a bubble of hot gas which bifurcates into two bubbles. These new bubbles are wafted out of the galaxy by gentle intergalactic winds and by their buoyancy in the cooler surrounding gas. The high external gas densities $\left(\sim 10^{-26} \mathrm{~g} \mathrm{~cm}^{-3}\right)$ needed to confine the bubble and provide sufficient buoyancy are likely to occur only in clusters of galaxies, if anywhere. Moreover, this gas must be very homogeneous and have very small turbulent motion if the bubble is to remain coherent. This model needs some detailed hydrodynamic calculations. For example, under what conditions does a bubble form rather than a galactic wind?

Galactic winds were discussed in Section I.2. While there does not seem to be any observational evidence for their existence, the general idea is certainly plausible. Violent hydrodynamic explosions could produce the phenomena in M82, for example. Such an explosion might be set off by the nuclear reactions in a supermassive star (Appenzeller and Fricke, 1972). In a non-magnetic non-rotating spheroid with an exponential density distribution, a point explosion at the centre tends to break out first along the main axes (Sakashita, 1971). However, this tendency is not pronounced unless the eccentricity of the spheroid is $\gtrsim 0.7$. Strong, global magnetic fields might focus the explosion into a jet, but such problems have not been calculated in detail.

Direct gravitational ejection can be more efficient than methods which first convert gravity to magnetic or rotational forces. Wheeler (1971) has described one such mechanism, the 'tube of toothpaste' instability. This may occur as a star falls into the ergosphere of a massive rotating black hole. The star becomes tidally elongated and disrupts. Some of its material manages to extract energy from the ergosphere and be ejected, perhaps in a manner resembling a jet. Simple models of this process (Mashhoon, 1973) suggest that, although the escaping pieces may leave with speeds $\sim 0.2 \mathrm{c}$, the efficiency of energy extraction is only $\sim 10^{-3}$ in physically reasonable cases.

There are two ways of producing gravitational ejection. One is by imparting enough kinetic energy to a particle so that it escapes. The other is by removing enough binding energy so that a particle can escape with its original kinetic energy. Processes which may cause a loss of binding energy are gravitational radiation, or the explosive expulsion of intergalactic gas. The effects of a sudden loss of binding energy in a cluster of particles are discussed by Field and Saslaw (1971), Aarseth and Saslaw (1972) and Case (1972). This mass release does not generally produce a preferred direction of ejection, so it is probably not relevant to most galactic nuclei, although it may be related to the virial mass discrepancy in clusters of galaxies. 
Perhaps the simplest method of gravitational ejection, indeed the simplest ejection process of any type, is the gravitational slingshot. This idea harkens back to Lagrange (1783) who noticed that the Newtonian 3-body problem is generally unstable. Consider three mass points interacting gravitationally with negative total energy. If the masses of two of these particles are much less than that of the third, the two will become relatively stable satellites of the most massive body, and nothing very interesting from the point of view of ejection is likely to happen. However, if the three bodies have masses and separations of the same order of magnitude, the system is rapidly unstable. Two of the objects can give enough kinetic energy to the third so that it escapes. Energy conservation requires that the two remaining objects from a compact binary, and linear momentum conservation requires the binary to move in the opposite direction to the ejected object.

Clearly then, if three or more massive objects can form (e.g. by fragmentation of gas clouds or by coalescence of colliding stars) in a galactic nucleus, the gravitational slingshot may eject them from the galaxy. In systems with many objects, numerical computations indicate that after several crossing-times the three most massive objects will tend to interact strongly at the centre in this way. To explore the gravitational slingshot in detail, Saslaw et al. (1974) computed some 25000 cases of three-body scattering and several hundred cases of four-body interaction, for a wide range of initial orbital parameters and mass ratios.

The main features of ejection by the gravitational slingshot are:

(1) the double configuration characteristic of many extragalactic radio sources follows from conservation of momentum, with one component of the source a single massive object and the other component a binary, provided only that the ejection velocities exceed the escape velocity from the parent galaxy or quasar.

(2) the numerical experiments indicate that the ejection velocities are likely to be relatively low $-\mathbf{a}$ few thousand $\mathrm{km} \mathrm{s}^{-1}$ rather than relativistic. There is some evidence for non-relativistic velocities (Mackay, 1973).

(3) the massive objects can account for compact components observed in many radio sources, without the need to invoke additional confinement mechanisms.

(4) the massive objects are nearly always ejected close $\left(\$ 20^{\circ}\right)$ to the plane of their total angular momentum. Thus if this plane is also approximately the plane of the galaxy (because the massive objects formed from material whose angular momentum was typical of the galaxy), the objects would be ejected roughly in the plane of the galaxy. This feature is especially important since the other uncontrived ejection mechanisms calculated in detail predict that matter escapes perpendicular to the galactic plane, approximately parallel to the polar axis along the path of least resistance. This can be checked observationally. Present evidence suggests that double radio sources tend to be ejected in the plane of their parent galaxy (Mackay, 1971; Bridle and Brandie, 1973). Some consequences of the passage of such massive objects through the interstellar medium of a galaxy are discussed by Saslaw and De Young (1972).

The problems of the gravitational slingshot approach to the structure of extragalactic radio sources are mainly to understand the formation and stability of the 
TABLE I

Properties of ejection processes

\section{Natural consequences}

$\begin{array}{ll}\text { Ejection } & \text { Primary } \\ \text { mechanism } & \text { ejecta }\end{array}$

Jet $^{\text {a }}$ Double Compact

symmetry radio

Ejection Much

relative to quantitative

components $^{\text {it }}$ polar axis calculation

\begin{tabular}{|c|c|c|c|c|c|}
\hline Magnetic wind up & gas & yes & yes & no & 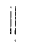 \\
\hline Magnetic flares & gas & yes & yes & no & \\
\hline Recoiling magnetoid & magnetoid & yes & $?$ & yes & $?$ \\
\hline Radiation pressure & gas & no & no & no & $\ldots$ \\
\hline Buoyant bubble & gas & $?$ & $?$ & no & $\|$ \\
\hline Galactic wind & gas & no & no & no & $\ldots$ \\
\hline $\begin{array}{l}\text { Hydrodynamic } \\
\text { explosion }\end{array}$ & gas & no & $?$ & no & \\
\hline Tube of toothpaste & gas, star bits & yes & $?$ & $?$ & $?$ \\
\hline Mass release & $\begin{array}{l}\text { compact objects, } \\
\text { gas }\end{array}$ & no & no & yes & $\ldots$ \\
\hline $\begin{array}{l}\text { Gravitational } \\
\text { slingshot }\end{array}$ & $\begin{array}{l}\text { compact massive } \\
\text { objects }\end{array}$ & yes & yes & yes & $\dot{L}$ \\
\hline
\end{tabular}

a without additional confinement mechanisms

massive objects, and their detailed radiation mechanisms. These properties are more complex than the ejection process, and they need careful investigation.

Table I summarizes some important aspects of these ejection mechanisms, including a rather subjective impression of whether the ejection process has been calculated in detail. From the observations it is clear that no one ejection process can operate universally. From the theories of the last few years we now know several possible ways to expel matter from the nuclei of galaxies. But the secret still sits in the middle. Perhaps by the next General Assembly of the IAU, the more likely mechanisms will be sorted out, and this ring of possibilities will start to change into an inward spiral.

Part of this review was written at the Aspen Center for Physics during the summer of 1973. It was completed during a visit to St Andrew's College of the University of Sydney, and I am happy to thank the Principal and Fellows of St Andrew's College for their friendly hospitality.

\section{References}

Aarseth, S. J. and Saslaw, W. C.: 1972, Astrophys. J. 172, 17.

Ambartsumian, V. A.: 1965, in The Structure and Evolution of Galaxies, Wiley Interscience, New York, p. 1.

Anand, S. P. S. and Shara, M. M.: 1972, Astrophys. Space Sci. 16, 171.

Andrillat, Y. and Souffrin, S.: 1971, Astron. Astrophys. 11, 286.

Appenzeller, I. and Fricke, K. J.: 1972, Astron. Astrophys. 21, 285.

Arny, T. T.: 1970, Monthly Notices Roy. Astron. Soc. 148, 63.

Arny, T. T.: 1971, Astrophys. J. 169, 289.

Arny, T. T. and Weissman, P.: 1973, Astron. J. 78, 309.

Arons, J., Gunn, J. E., Kulsrud, R. M., and Ostriker, J. P.: 1974, in preparation.

Arp, H. and Bertola. F.: 1970, Astrophys. Letters 6, 65. 
Bardeen, J. M.: 1970, Nature 226, 64.

Bardeen, J. M., Press, W. H., and Teukolsky, S. A.: 1972, Astrophys. J. 178, 347.

Bergeron, J. and Salpeter, E. E.: 1973, Astron. Astrophys. 22, 385.

Bisnovatni-Kogan, G. S. and Sunyaev, R. A.: 1972, Soviet Astron. 15, 697.

Blandford, R. D. and Rees, M. J.: 1972, Astrophys. Letters 10, 77.

Bonometto, S. A. and Saggion, A.: 1973, Astrophys. Letters 13, 193.

Bridle, A. H. and Brandie, G. W.: 1973, Astrophys. Letters 15, 21.

Burbidge, G. R.: 1970, Ann. Rev. Astr. Astrophys. 8, 369.

Burbidge, E. M. and Burbidge, G. R.: 1965, Astrophys. J. 142, 1351.

Burbidge, E. M. and Burbidge, G. R.: 1971, Astrophys. J. Letters 163, L21.

Burke, J. A.: 1968, Monthly Notices Roy. Astron. Soc. 140, 241.

Case, L. A.: 1972, Astrophys. J. 173, 665.

Cavaliere, A., Morrison, P., and Wood, K.: 1971, Astrophys. J. 170, 223.

Chertoprud, V. E., Gudzenko, L. I., and Ozernoy, L. M.: 1973, Astrophys. J. Letters 182, L53.

Colgate, S. A.: 1967, Astrophys. J. 150, 163.

Daltabuit, E. and Cox, D.: 1972, Astrophys. J. Letters 173, L13.

Davidson, K.: 1972, Astrophys. J. 171, 213.

Dent, W. A.: 1972a, Astrophys. J. Letters 175, L55.

Dent, W. A.: 1972b, Science 175, 1105.

de Sabbata, V., Fortini, P., and Gualdi, C.: 1972, Astrophys. Letters 12, 87.

De Young, D. S.: 1968, Astrophys. J. 153, 633.

De Young, D. S.: 1971, Astrophys. Letters 9, 43.

De Young, D. S., Roberts, M. S., and Saslaw, W. C.: 1974, Astrophys. J. 185, 809.

Disney, M.: 1973, Astrophys. J. Letters 181, L55.

Disney, M. J. and Cromwell, R. H.: 1971, Astrophys. J. Letters 164, L35.

Downes, D. and Martin, A. H. M.: 1971, Nature 233, 112.

Field, G. B. and Saslaw, W. C.: 1971, Astrophys. J. 170, 199.

Fricke, K. J.: 1973, Astrophys. J. 183, 941.

Frost, R.: 1951, 'The Secret Sits' in Complete Poems, Jonathan Cape, London, p. 394.

Gerlach, U. H.: 1971, Astrophys. J. 168, 481.

Gull, S. F. and Northover, K. J. E.: 1973, Nature 244, 80.

Hansen, R. O.: 1971, Astrophys. J. 170, 557.

Harrison, E. R.: 1971, Monthly Notices Roy. Astron. Soc. 154, 167.

Heeschen, D. S., Morgan, W. W., and Walborn, N. R.: 1971, Astrophys. J. Letters 165, L65.

Hoyle, F. and Fowler, W. A.: 1963a, Monthly Notices Roy. Astron. Soc. 125, 169.

Hoyle, F. and Fowler, W. A.: 1963b, Nature 197, 533.

Hoyle, F. and Narlikar, J. V.: 1966, Proc. Roy. Soc. A. 290, 177.

Illarionov, A. F. and Sunyaev, R. A.: 1972, Astrophys Space Sci. 19, 61.

Jeans, J. H.: 1929, Astronomy and Cosmogony, Cambridge University Press, p. 352.

Johnson, H. E. and Axford, W. I.: 1971, Astrophys. J. 165, 381.

Jones, T. W. and Kellogg, P. J.: 1972, Astrophys. J. 172, 283.

Kaneko, N., Toyama, K., and Nichimuna, M.: 1972, Astrophys. Space Sci. 18, 121.

Kardashev, N. S.: 1970, Sov. Astron. 14, 375.

Kellermann, K. I., Jauncey, D. L., Cohen, M. H., Shaffer, B. B., Clark, B. G., Broderick, J.,

Rönnäng, B., Rydbeck, O. E. H., Matveyenko, L., Moiseyev, I., Vitkevitch, V. V., Cooper,

B. F. C., and Batchelor, R.: 1971, Astrophys. J. 169, 1.

Krishna Swamy, K. S.: 1971, Astrophys. J. 167, 63.

Kronberg, P. P., Pritchet, C. J., and van den Bergh, S.: 1972, Astrophys. J. Letters 173, L47.

Kunkel, W. E. and Bradt, H. V.: 1971, Astrophys. J. Letters 170, L7.

Lagrange, J.: 1873, in M. J. A. Serret (ed.), Oeuvres, vol. 6, Gauthier-Villars, Paris.

Landstreet, J. P. and Angel, J. R. P.: 1972, Astrophys. J. Letters 174, L127.

Larson, R. B.: 1969, Monthly Notices Roy. Astron. Soc. 145, 405.

Levich, E. V. and Sunyaev, R. A.: 1971, Soviet Astron. 15, 363.

Levich, E. V., Sunyaev, R. A., and Zeldovich, Ya. B.: 1972, Astron. Astrophys. 19, 135.

Lynden-Bell, D. and Rees, M. J.: 1971, Monthly Notices Roy. Astron. Soc. 152, 461.

Lynds, C. R.: 1970, Astrophys. J. Letters 159, L151.

MacAlpine, G. A.: 1972, Astrophys. J. 175, 11. 
Mackay, C. D.: 1971, Monthly Notices Roy. Astron. Soc. 151, 421.

Mackay, C. D.: 1973, Monthly Notices Roy. Astron. Soc. 162, 1.

Mashhoon, B.: 1973, Astrophys. J. Letters 181, L65.

Mathews, W. G.: 1972, Astrophys. J. 174, 101.

Mathews, W. G. and Baker, J. C.: 1971, Astrophys. J. 170, 241.

Mathis, J. S.: 1967, Astrophys. J. 147, 1050.

Milne, E. A.: 1926, Monthly Notices Roy. Astron. Soc. 86, 459.

Mushotzky, R. F., Solomon, P. M., and Strittmatter, P. A.: 1972, Astrophys. J. 174, 7.

Ne'eman, Y. and Tauber, G.: 1967, Astrophys. J. 150, 755.

Norman, C. A. and ter Haar, D.: 1973, Astron. Astrophys. 24, 121.

Novikov, I. D.: 1965, Soviet Astron. 8, 857.

Novikov, I. D. and Thorne, K. S.: 1972, Les Houches Lectures.

O'Connell, D. J. K. (ed.): 1971, Nuclei of Galaxies, North-Holland Publ. Co., Amsterdam.

Ozernoy, L. M.: 1973, Soviet Astron. 16, 916.

Ozernoy, L. M. and Somov, B. V.: 1971, Astrophys. Space Sci. 11, 264.

Ozernoy, L. M. and Usov, V. V.: 1971, Astrophys. Space Sci. 13, 3.

Ozernoy, L. M. and Usov, V. V.: 1973a, Astrophys. Letters 13, 209.

Ozernoy, L. M. and Usov, V. V.: 1973b, Astrophys. Space Sci. 25, 149.

Pacholczyk, A. G.: 1970, Astrophys. J. Letters 161, L207.

Peebles, P. J. E.: 1972, Astrophys. J. 178, 371.

Penston, M. V., Penston, M. J., Neugebauer, G., Tritton, K. P., Becklin, E. E., and Visvanathan, N.: 1971, Monthly Notices Roy. Astron. Soc. 153, 29.

Press, W. H. and Thorne, K. S.: 1972, Ann. Rev. Astron. Astrophys. 10, 335.

Quirk, W. J. and McKee, C. F.: 1971, Astrophys. J. 169, 119.

Rees, M. J.: 1971, Nature 229, 312.

Rees, M. J., Silk, J. I., Werner, M. M., and Wickramasinghe, N. C.: 1969, Nature 223, 788.

Rieke, G. H. and Low, F. J.: 1972a, Astrophys. J. Letters 176, L95.

Rieke, G. H. and Low, F. J.: 1972b, Astrophys. J. Letters 177, L115.

Roxburgh, I. W. and Saffman, P. G.: 1965, Monthly Notices Roy. Astron. Soc. 129, 181.

Ryan, M. P.: 1972, Astrophys. J. Letters 177, L79.

Sakashita, S.: 1971, Astrophys. Space Sci. 14, 431.

Salpeter, E. E. and Wagoner, R. V.: 1971, Astrophys. J. 164, 557.

Sanders, R. H.: 1970, Astrophys. J. 162, 791.

Saslaw, W. C.: 1973, Publ. Astron. Soc. Pacific 85, 5.

Saslaw, W. C. and De Young, D. S.: 1972, Astrophys. Letters 11, 87.

Saslaw, W. C., Valtonen, M. J., and Aarseth, S. J.: 1974, Astrophys. J. 190, 253.

Scargle, J. D.: 1973, Astrophys. J. 179, 705.

Scharlemann, E. T. and Wagoner, R. V.: 1972, Astrophys. J. 171, 107.

Schwarzschild, M.: 1973, Astrophys. J. 182, 357.

Sciama, D. W. and Rees, M. J.: 1967, Nature 216, 147.

Seidle, F. G. P. and Cameron, A. G. W.: 1972, Astrophys. Space Sci. 15, 44.

Shapiro, S. L.: 1973, Astrophys. J. 180, 531

Shklovsky, J.: 1970, Nature 228, 1174.

Spitzer, L.: 1942, Astrophys. J. 95, 329.

Spitzer, L.: 1971, in D. J. K. O'Connell (ed.), Nuclei of Galaxies, North-Holland Publ. Co., Amsterdam, p. 443.

Spitzer, L. and Saslaw, W. C.: 1966, Astrophys. J. 143, 400.

Spitzer, L. and Stone, M. E.: 1967, Astrophys. J. 147, 519.

Steigman, G. and Strittmatter, P. A.: 1971, Astron. Astrophys. 11, 279.

Stockton, A.: 1972, Astrophys. J. 173, 247.

Sturrock, P. A.: 1971, Astrophys. J. 170, 85.

Sturrock, P. A. and Barnes, C.: 1972, Astrophys. J. 176, 31.

Tovmassian, H. M.: 1972, Astrophys. J. Letters 178, L47.

van der Kruit, P. C.: 1971, Astron. Astrophys. 15, 110.

Wade, C. M. et al.: 1971, Astrophys. J. Letters 170, L11.

Wheeler, J. A.: 1971, in D. J. K. O'Connell (ed.), Nuclei of Galaxies, North-Holland Publ. Co., Amsterdam, p. 560. 
Williams, R. E.: 1972, Astrophys. J. 178, 105.

Wilson, J. R.: 1972, Astrophys. J. 173, 431.

Wolfe, A. M.: 1974, Astrophys. J. 188, 243.

Wolfe, A. M. and Burbidge, G. R.: 1970, Astrophys. J. 161, 419.

Zel'dovich, Ya. B. and Novikov, I. D.: 1971, Relativistic Astrophysics, Univ. of Chicago Press.

\section{DISCUSSION}

G. de Vaucouleurs: I am surprised that you have not discussed the effects of capture of infalling material by galactic nuclei.

Saslaw: I did not do so because I considered that such matter would already have formed the main constituents of the nuclei, but I agree that it's an important process to be considered in the detailed evolution of nuclei since it may alter the chemical composition.

E. M. Burbidge: I have a comment concerning radiation-driven gas outflow from nuclei of galaxies and QSOs and the way in which radiation pressure outwards can balance gravity acting inwards. Such a balance depends on constancy of light, but in NGC 4151 the He I absorption lines are variable (the triple structure observed at Lick charged in a time $\sim 1 \mathrm{yr}$ ) and its nuclear light is also variable. None of the QSOs with multiple absorption line redshifts are known to be variable, and the absorption lines have remained visible in some for a few years. It would be interesting to know to what level one can say their light is constant: smallish variations should destroy the balance and the absorption systems should change.

Saslaw: Your first point about the association between constant luminosity and constant velocity in the absorption lines is an important one, and NGC 1275 would be an especially interesting case to study. However, we should keep in mind that the luminosity of these objects may well come from several sources in the nucleus, and not all these sources need have constant luminosity. Thus while a positive correlation between constant luminosity and multiple absorption line systems would be significant, lack of correlation would not provide a strong test if there are multiple sources of luminosity and some of them vary.

E. M. Burbidge: You seemed to suggest that galactic winds flowing outward are a necessary consequence of stellar evolution in the central region. Wouldn't this conflict with the infall of gas postulated to form new stars in the nuclei in dense configurations?

Saslaw: The conditions necessary for galactic winds to occur depend on the temperature and density of the interstellar gas and there wasn't time to describe this in detail. Roughly, if the temperature is high enough $\left(\gtrsim 10^{6} \mathrm{~K}\right)$, a wind may flow out of the nucleus. For a low temperature $\left(\lesssim 10^{4} \mathrm{~K}\right.$ ), pressure support is insufficient and the gas flows into the nucleus. At intermediate temperatures the situation is especially complex since the gas is prone to thermal and hydrodynamic instabilities. The exact dependence on temperature and density, of course, has to be found from the detailed models, but there seems to be a regime of inflow and a regime of outflow.

Arp: With respect to the gravitational sling-shot model, it is interesting to note that the companions on the ends of spiral arms - which I argued had been ejected from the nucleus in the plane - that some of these companions are on the end of curiously doubled spiral arms. From what you just said the sling-shot model would give a double ejection in one of the two opposite directions.

Saslaw: The separation of the double would be extremely small, perhaps only $1 / 100 \mathrm{pc}$ or less.

Arp: Yes, but it would probably be unstable and come apart.

G. Burbidge: Suppose strange things are happening (such as Arp was suggesting this morning) and I'll take money on that, how would your ideas be affected?

Saslaw: If it were indeed the case that nuclei are ejecting other, very massive, compact objects then a knowledge of the velocities would be important. I think the sling-shot idea is in this case the only feasible one which has been looked at in detail. Newtonian calculations indicate velocities of a few thousand kilometres per second, and I don't know whether detailed relativistic calculations would predict relativistic velocities or not. There may then be the problem of blue-shifts, not yet observed. That's one I'll leave to you.

G. Burbidge: That's an almost insuperable difficulty.

G. de Vaucouleurs: I notice that most of your proposed mechanisms imply ejection of matter parallel to the rotation axis. This brings to mind the explosion in M82 and also a study by Holmberg (Arkiv Astron. 5, 305. 1969) of the angular distribution of companions of galaxies seen on edge, where he found a significant excess of companions near the minor axis. 
Saslaw: We should bear in mind that several of these mechanisms may be operating and it may be dangerous to try to account for everything in terms of one mechanism; history has shown that the Universe is complex. One of the interesting questions relating to this is whether the components of double radio sources associated with elliptical galaxies tend to lie along the major or the minor axes of the galaxies. There have been two studies, one by Mackay (Monthly Notices Roy. Astron. Soc. 151, 421, 1971) and one by Bridle and Brandie (Astrophys. Letters 15, 21, 1973). The statistics are small and it seems to be too early to draw firm conclusions from their results, but the indication is that the components are preferentially aligned with the major axes, i.e. with the planes of the galaxies rather than the rotation axes. It is most important to improve these statistics, since it is possible in this way to decide between the different mechanisms of ejection.

Oort: In considering nuclei of galaxies it is useful to look at the nucleus of our own Galaxy, which is one that we can observe in considerably greater detail. The infrared observations by Rieke and Low show a structure more complex than has been considered in Dr Saslaw's communication. There are five or more distinct patches of diameter $\frac{1}{4} \mathrm{pc}$ or less, all contained in a region of about 1 pc diameter. The observations of the $\mathrm{CO}$ emission around $\mathrm{Sgr} \mathrm{A}$ and $\mathrm{Sgr} \mathrm{B} 2$ indicate something else that is unexpected, viz. a 'wind' with a velocity of about $50-80 \mathrm{~km} \mathrm{~s}{ }^{1}$ apparently blowing past these two sources and having a rather steep velocity gradient.

G. de Vaucouleurs: In barred spirals such as NGC 1365 one also gets the impression of a flow through the nucleus.

Sargent: One mechanism you mentioned for forming quasars is to have stellar collisions in very dense stellar systems in the nuclei of galaxies. Now the densest stellar systems we know of, such as the

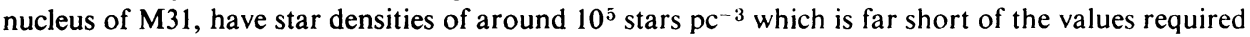
by the stellar collision hypotheses. Have you given any thought to whether systems which are nearly dense enough to become active could be detected, for example in the compact galaxies?

Saslaw: In principle it should be possible to detect such systems. The main problem (apart from getting the necessary resolution) is to look for systems where most of the nuclear radiation is still thermal so that the presence of stars could be established more directly.

Rickard: One has to be careful about observations of concentrations in the nuclei of galaxies. I've taken photographs in Chile, where the seeing is occasionally very gcod, of some of the 'hot spots' pointed out by Dr de Vaucouleurs in southern galaxies, and resolved them into chains of H II regions. So there is nothing unusual about these particular irregular concentrations.

van den Bergh: Inspection of plates of NGC 4151 shows that the Seyfert nucleus is embedded in a large disk with a rather pathological structure. The fact that this structure extends to a large distance from the nucleus suggests that the nuclear disturbance has been going on for a considerable time.

Saslaw: Is there any evidence that it might have been going on in spurts but not continuously?

$G$. Burbidge: How many galaxies have been looked at in such detail as NGC 4151, so that one can be sure it really is pathological?

van den Bergh: I think NGC 4151 is quite unique.

G. Burbidge: This contradicts to some extent what was said earlier, i.e. that Seyfert galaxies are fairly normal systems outside their nuclei.

van den Bergh: NGC 4151 is the only case I know of where the outer structure is so peculiar.

G. de Vaucouleurs: It would be very important to know whether Seyfert galaxies are entirely normal outside the nucleus or whether there are features outside the nucleus which may be associated with the nuclear activity. Some authors have recently drawn attention to the outer ring in NGC 1068 as a possible example. Now, of the 12 classical Seyfert galaxies, 3 (NGC 1068, 3516, 7469) have outer ring structures. This may seem at first sight possibly significant until one realises that Seyferts are confined to a rather narrow range in the Hubble sequence, from intermediate lenticulars to Sbc, and that about one-quarter of all galaxies in this range have an outer ring structure.

Heidmann: From the point of view of neutral hydrogen radiation, Seyfert galaxies are not different from normal galaxies, either when looking at the relative amount of neutral hydrogen or its velocity distribution (ref. Astron. Astrophys. 6, 453, 1970).

Disney: I would like to ask Dr Ulrich if there is a real continuity between Seyfert and elliptical nuclear spectra. I have the impression that you do not find strong optical nuclei in ellipticals and that the line widths are nowhere near so broad.

Ulrich: I agree. 
Ekers: Since the observations of extended radio sources associated with QSOs argue strongly for QSOs being in the nuclei of E galaxies, it seems surprising that the optical spectra of the QSOs resemble those of the nuclei of Seyfert galaxies, which are spirals, more than they do those of ellipticals.

Ulrich: It does not seem that there is any tight correlation between the amounts of energy released in various ways: for example, the infrared luminosity is not correlated with the X-ray luminosity or with the width of the hydrogen lines.

van Woerden: If Seyferts are cores of spirals and quasars are cores of ellipticals, can there be a transition between Seyferts and quasars, just as there is a transition between spirals and ellipticals?

G. Burbidge: The answer to that is clearly yes.

G. de Vaucouleurs: (i) Seyferts are spirals or lenticulars, not just cores.

(ii) There have been suggestions that Seyfert nuclei may be related to quasars.

(iii) There is no evidence of a transition between spirals and ellipticals, except in a classificatory sense, lenticulars being placed between ellipticals and spirals; no evolution along the sequence is implied and there seems to be a discontinuity between lenticulars and ellipticals.

Heidmann: Yes, I would like to emphasize this point: the relative amount of neutral hydrogen in ellipticals is much smaller than one would expect from an extrapolation of its variation along the Hubble sequence from irregulars down to lenticulars; there is a break in the sequence between ellipticals and lenticulars (Astron. Astrophys. 25, 451, 1973).

Freeman: Ekers points out how double radio sources occur in elliptical galaxies only. Many lenticulars appear mainly elliptical in the sense that they have dominant bulge components. It would be interesting to observe some lenticulars in the continuum, to see if they look more like ellipticals than spirals (assuming they radiate at all in the continuum).

Larson: l'd like to make a comment in favour of open-mindedness in the interpretation of anomalies in the outer structure of galaxies with active nuclei. People have generally tried to interpret such anomalies as being a result of the activity in the nucleus, but it is also possible that in some cases the anomalous outer structure and the nuclear activity may have a common cause connected with the overall dynamics or evolution of the galaxy. For example, if nuclear activity has anything to do with condensation of matter into the nucleus, it may be that the structural anomalies could be interpreted as manifestations of the same condensation process on a larger scale. Either the infall of intergalactic gas or the continuing condensation of a remnant protogalactic envelope could be a possible cause of such a situation.

G. Burbidge: Are you thinking in terms of accretion from outside, or processes which conserve mass in a galaxy?

Larson: Perhaps just condensation of gas that's already in a galaxy or near it, such as the neutral hydrogen halos round M81 and M82.

G. Burbidge: There is no evidence for $\mathrm{H}$ I near ellipticals, which are sometimes the seat of violent activity, and it's hard to argue in these cases that the activity is due to gas. It must be attributed to stars in the nucleus or to something else.

Osmer: At Cerro Tololo, M. Smith, D, Weedman and I have been making spectrophotometric observations of the southern galaxies with peculiar nuclei which were found by Sersic and Pastoriza, as well as the Seyferts NGC 1566 and 3783. The former galaxies have luminosities in $\mathrm{H} \beta$ that in many cases are as large as are found in Seyfert galaxies, although the gaseous regions are also much larger in physical extent. NGC 3783 is a barred spiral with an apparently normal Seyfert-type nucleus, while NGC 1566 has the lowest $\mathbf{H} \beta$ luminosity yet known, its spectrum otherwise being similar to that of NGC 1058. The Balmer lines had widths of several thousand $\mathrm{km} \mathrm{s}^{-1}$ in January 1973.

Freeman: We have a collection of spectra of the nucleus of the ex-Seyfert galaxy NGC 1566. In $1956 \mathrm{H} \beta$ was about $3000 \mathrm{~km} \mathrm{~s}^{-1}$ wide (de Vaucouleurs). In $1968 \mathrm{H} \beta$ was clearly double - one component had the same redshift as the forbidden lines and the other was about $800 \mathrm{~km} \mathrm{~s}^{-1}$ to the red. By March $1972 \mathrm{H} \beta$ was very sharp, rather weaker, and single, so the spectrum could not be described as Seyfert-like.

Cox: Infall of material in a galaxy can proceed at low densities and high temperature until a rapid cooling occurs near the centre, bringing about a high density contrast. It seems to me that a very modest infall over a long period of time can cause a large accumulation of matter in the nucleus. Thus we might easily end up with any of several of the dramatic types of objects Saslaw has out-lined.

G. Burbidge: Shklovsky (Astron. Zh. 39, 591, 1962) considered this possibility for the radio source M87 some years ago, but the necessary accretion rates were very high.

Cox: I do not suggest that such a modest infall could sustain the required power output in a 
steady state, but could slowly generate an object which would then be dramatic for a limited period of time.

Larson: I don't think that the suggested role of infall or condensation processes as a contributing cause of nuclear activity is necessarily inconsistent with the absence of any clear evidence of this process in many galaxies showing nuclear activity. One should distinguish two stages in time: an earlier stage when gas inflow proceeds vigorously and is the dominant hydrodynamic process, and a later stage when a sufficient amount of mass has gone into pulsars (or other energy sources) to produce intense quasar activity. By this later time the infall process may have diminished in intensity, and in any case it is likely that the quasar activity itself might tend to reverse the inflow and disperse much of the residual gas in and around the galaxy. Thus by the time a spectacular object (e.g. quasar) has been produced, the dominant hydrodynamic process may be outflow rather than inflow. Perhaps there is an analogy with the formation of a massive $O$ star: by the time a newborn $O$ star has become visible, the initial collapse process has been terminated and one observes predominantly the gas outflows associated with the development of an expanding $\mathrm{H}$ II region.

Tiff: In Virgo, nuclear peculiarities seem to concentrate in the centre of the cluster - perhaps where the concentration of intergalactic material is higher. This is in interesting contrast with Coma where peculiarities like emission lines are found mainly in the outer parts of the cluster.

Miley: New measurements with the Westerbork telescope of the structure of NGC 1275 show that there is a component of size $\sim 30^{\prime \prime}$ to $1^{\prime}$, comparable with the size of the optical galaxy. The flux density amounts to about $2 \times 10^{-26} \mathrm{~W} \mathrm{~m}^{-2} \mathrm{~Hz}^{-1}$ at $1.4 \mathrm{GHz}$ and $\mathrm{I}$ wonder if emission from this source could be partially responsible for the new $\mathrm{H}_{\mathrm{I}}$ absorption results which you reported.

Saslaw: It may be related. 